Fidalgo, C., Collado, S. \& Senís, J. (2019). Del simulacro a la realidad: mejora del rendimiento de los estudiantes ante el TFM a través de un proyecto de innovación docente. Revista Electrónica Interuniversitaria de Formación del Profesorado, 22(3), 157-171.

DOI: http://dx.doi.org/10.6018/reifop.22.3.357421

\title{
Del simulacro a la realidad: mejora del rendimiento de los estudiantes ante el TFM a través de un proyecto de innovación docente
}

\author{
Camino Fidalgo, Silvia Collado, Juan Senís \\ Universidad de Zaragoza
}

\section{Resumen}

El TFM es una tarea difícil para los estudiantes de Máster, que se enfrentan a él con ansiedad, poca autoeficacia y sin las competencias necesarias para su correcta realización. Teniendo esto en cuenta, nuestro trabajo refleja los resultados de un proyecto de innovación docente destinado a lograr un mejor rendimiento en la redacción y defensa oral del TFM. Dicho programa se compone de cinco seminarios teórico-prácticos que cubren las competencias clave escritas y orales que debía adquirir el alumnado para la superación del TFM. Siguiendo un diseño experimental pre-post intervención, se evaluó la percepción de eficacia y el nivel de ansiedad de los alumnos. Además, se examinó el rendimiento del alumnado en un simulacro escrito de TFM y su posterior defensa oral. Los resultados muestran que existen diferencias estadísticamente significativas en todos los aspectos evaluados. Por un lado, se obtuvieron calificaciones más altas en el TFM respecto a las obtenidas por los estudiantes del curso anterior que no habían participado en el proyecto. Por otro lado, los participantes se consideraron más eficaces en la escritura y la defensa oral del TFM al finalizar el programa, al tiempo que se redujeron sus niveles de ansiedad.

\section{Palabras clave:}

Autoeficacia; ansiedad; habilidades escritas; habilidades comunicativas

\section{Contacto:}

Silvia Collado, scollado@unizar.es, Universidad de Zaragoza, Edificio de Bellas Artes, Ciudad Escolar sn 44003 Teruel.

Fuentes de financiación: Vicerrectorado de Política Académica de la Universidad de Zaragoza (PIIDUZ_17_047)”.Gobierno de Aragón (Departamento de Innovación, Investigación y Universidad) y FEDER “Construyendo Europa desde Aragón” para los grupos de investigación S31_17D, S24_17R y S45_17D. 


\title{
From Simulation to Reality: Improvement of Student Performance in the TFM through a Teaching Innovation Project
}

\begin{abstract}
Master's Thesis (TFM) is a difficult task for Master students. They face the writing and oral examination with anxiety, low levels of self-efficacy and a general lack of the necessary competencies for its correct execution. Our study reflects the results of a teaching innovation project aimed at reducing students' anxiety and increasing their self-efficacy to face their TFM. Five theoretical-practical seminars were designed to improve student's competences related to academic-scientific skills, both oral and writing ones. Through a pre-post experimental procedure, we evaluated students' self-efficacy and levels of anxiety towards their TFM. In addition, students' performance has been examined through a simulation of TFM and its subsequent oral defense. Our results show statistically significant differences between pre and post-intervention in all the aspects evaluated in the program. On one hand, participants obtained in their TFM when compared with the students of previous years which did not take part in this project. On the other hand, participants were more successful in completing their TFM and showed lower levels of anxiety after taking the seminars.
\end{abstract}

\section{Key words:}

Self-efficacy; anxiety; writing skills; oral skills

\section{Introducción}

La introducción de asignaturas en el Grado y en el Máster relacionadas con la investigación, tales como el TFG y el TFM, muestra la importancia de formar a los alumnos de los estudios de Grado de Magisterio y del Máster de Secundaria en la metodología de la investigación (Tejedor Tejedor, 2018), pero a su vez exige la adquisición por parte del alumno de competencias relacionadas con la lectura y escritura académico-científica, así como con la exposición y defensa oral de sus trabajos. La escritura académico-científica implica que, por un lado, el alumno actúe como investigador y, por otro, logre comunicar su trabajo de forma adecuada (Castelló e Iñesta, 2012). Partiendo de esta base, el presente trabajo aborda el bajo rendimiento de los alumnos del Máster de profesorado en el desarrollo del TFM de forma integral. Para ello se tiene en cuenta la autoeficacia y el nivel de ansiedad del alumnado ante la redacción y defensa oral del TFM, y cómo estas variables se relacionan con el rendimiento que muestra el alumno tanto de la escritura académico-científica como de exposición oral.

Escribir utilizando un estilo académico-científico es una tarea compleja que requiere el manejo de competencias rara vez adquiridas en otras asignaturas del Grado/Máster, tales como revisar literatura científica, e integrar y sintetizar lo leído, lo que incrementa la carga de trabajo del tutor académico a la hora de guiar al estudiante (Jato Seijas, Cajide Val, García Antelo y Zamora Rodríguez, 2018). Tanto es así que redactar en un estilo científico, de ágil lectura, con bibliografía relevante y sin errores gramaticales (Bhargava, 2015) es considerada una de las competencias más difíciles de adquirir durante los estudios de Grado y Máster (Pelias, 2003). 
Al igual que ocurre con la redacción de trabajos académicos, los estudiantes tampoco han adquirido formación específica para presentar y defender oralmente su trabajo (Chan, 2011). Este hecho se contrapone a la doble finalidad que para los estudiantes del Máster de profesorado tiene el adquirir competencias que les permitan ser buenos oradores. Por un lado, es relevante de cara a superar con éxito la defensa del TFM y supone un valor añadido al trabajo escrito que permite al estudiante resaltar la calidad de dicho trabajo (Blanco, 2009). Por otro, es importante para desarrollarse como futuros docentes, ya que gran parte de su trabajo se basa en transmitir de forma clara un mensaje, favoreciendo el proceso de enseñanza-aprendizaje (Sanjuán, Senís y Del Moral, 2017; Becerra Traver, 2017). Esta falta de competencia para la redacción y defensa oral de trabajos científicos da lugar a una percepción de ansiedad y de ineficacia por parte del alumnado para superar con éxito la asignatura TFM.

La autoeficacia es la capacidad percibida de hacer frente a situaciones concretas, y por lo tanto está referida a una tarea específica (Bandura, 1986).Numerosos estudios muestran la existencia de una relación positiva entre autoeficacia y rendimiento académico (DoménechBetoret, Abellán-Roselló, y Gómez-Artiga, 2017; Galleguillos, 2017; Lane y Lane, 2001; Pajares, 2001). Este hecho tiene una serie de implicaciones tanto en la redacción de trabajos académicos como en las exposiciones y defensas orales de los mismos. Por ejemplo, una alta autoeficacia conlleva no evitar situaciones difíciles (Roses y Humanes, 2014), algo que sí ocurre con muchos estudiantes que no presentan el TFM en primera convocatoria o que incluso lo retrasan años. Por otro lado, la autoeficacia se relaciona negativamente con la ansiedad. Así, cuanto menos autoeficaz se percibe un estudiante ante una tarea, mayor es su ansiedad (Contreras et al. 2005; Pervin, 1997). En línea con estos resultados, diversos estudios muestran la existencia de una relación positiva entre la autoeficacia de los alumnos y su rendimiento en la redacción de trabajos (Callinan, van der Zee y Wilson, 2018; Pajares y Johnson 1996). En este sentido, la redacción de un TFM, trabajo que va a ser evaluado por un tribunal, constituye una fuente importante de ansiedad y estrés para el estudiante (Bartlett y Mercer, 2000), sobre todo si éste cree no tener las competencias necesarias para poder escribirlo (Bazrafkan, Shokrpour, Yousefi y Yamani, 2016).

Por otro lado, la autoeficacia y la ansiedad también se han estudiado en relación con las habilidades comunicativas. Diversos estudios muestran cómo hablar en público produce ansiedad a gran número de personas (Orejudo, Fernández Turrado y Briz, 2012; Sánchez, Rosales, y Cañedo, 1994), traduciéndose en cambios fisiológicos (Onieva-López, 2016; Palatini et al., 2011). Además, existe una relación directa entre la ansiedad de hablar en público y la auto-imagen. Así, tras haber realizado una presentación oral, sujetos con una autoimagen negativa consideran haber ejecutado dicha presentación peor y se muestran más ansiosos que aquellos cuya autoimagen es positiva (Hirsch, Mathews, Clark, Williams y Morrison, 2006).

\section{Objetivos}

Los objetivos de este trabajo son investigar si la participación en un proyecto de innovación docente destinado a los alumnos del Máster de profesorado: produce una mejora en las habilidades de redacción de un trabajo académico similar al TFM (Objetivo 1), en las habilidades comunicativas (Objetivo 2) y en los niveles de autoeficacia y ansiedad, tanto en relación a las habilidades de escritura como de comunicación oral (Objetivo 3). Además, se estudia si los resultados obtenidos en la defensa del TFM son significativamente mejores en el curso 2017/2018 respecto al curso anterior 2016/2017 en cuanto a calificación obtenida por los estudiantes y número de TFM presentados en la convocatoria ordinaria (Objetivo 4). 


\section{Metodología}

\section{Población y muestra}

Los participantes fueron 29 estudiantes del Máster de profesorado que dieron su consentimiento informado. De éstos, 16 estudiantes del Máster de profesorado del curso 2017/2018 participaron en el proyecto de innovación docente y 13 estudiantes que cursaron el mismo Máster en el curso 2016/2017 formaron el grupo control. Las calificaciones obtenidas en el TFM de los alumnos del grupo control fueron necesarias para comparar si se produjo una mejoría en el rendimiento del TFM tras la participación en el proyecto.

\section{Instrumento}

Se impartieron cinco seminarios teórico-prácticos de 1 hora de duración durante el curso 2017/18 basados en las competencias a adquirir por los alumnos marcadas por la guía docente del TFM y divididas en competencias escritas y orales. Los seminarios se organizaron de la siguiente manera:

Seminario 1. Seminario teórico introductorio sobre el TFM: tipos y estructura de TFM, uso de registro académico, síntesis de ideas, y competencias necesarias para superar con éxito la asignatura.

Seminario 2. Seminario teórico introductorio sobre habilidades comunicativas orales: exposición de un trabajo científico, aspectos pragmáticos del discurso y su adecuación al contexto y al receptor, síntesis y uso pertinente de instrumentos complementarios visuales y coherencia y cohesión del discurso.

Seminario 3. Seminario teórico-práctico sobre búsqueda bibliográfica, selección de información y uso de la normativa APA.

Seminario 4. Seminario teórico-práctico sobre plagio: qué es el plagio académico y cómo evitarlo.

Seminario 5. Seminario teórico-práctico sobre habilidades de comunicación. Adecuación del discurso a un tribunal, lenguaje verbal y no verbal, adecuación al tiempo de exposición, argumentación de preguntas y comentarios del tribunal y no redundancia entre discurso y apoyo visual.

Las medidas utilizadas para registrarla autoeficacia, la ansiedad y el rendimiento son:

a) Autoeficacia en redacción de TFM. Se utilizó una escala compuesta por 5 ítems (véase Tabla 1), con una escala de respuesta tipo Likert desde 1 (no puedo) a 5 (estoy seguro de que puedo hacerlo). Por ejemplo: "Puedo: 1. Buscar fuentes bibliográficas con rigor científico (actualizadas, en castellano e inglés, contrastadas, etc.). La fiabilidad interna fue a To= .70 y a $11=.70$.

b) Ansiedad ante la redacción de TFM. Se utilizaron los mismos 5 ítems que en la escala anterior y con la misma escala de respuesta, pero haciendo referencia al grado de ansiedad que estas situaciones producirían a los alumnos, desde 1 (no me produciría ninguna ansiedad) hasta 5 (me generaría mucha ansiedad). La fiabilidad interna fue $\alpha$ To $=.88$ y a T1= .78 .

c) Rendimiento en la redacción del TFM. Escala de evaluación de los simulacros escritos de TFM (pre y post intervención), utilizada tanto por el docente para dar retroalimentación al alumno (Rendimiento redacción) como por los alumnos para co-evaluar el trabajo de un compañero en To. Consta de 9 ítems con valor de o a 1 (véase Tabla 3), siendo la calificación final la suma de la puntuación en los 9 ítems, sobre una escala de 0 a 5.

d) Autoeficacia ante la defensa oral del TFM. Se utilizó una escala compuesta por 3 ítems con escala de respuesta tipo Likert desde 1 (no puedo) a 5 (estoy seguro de que puedo hacerlo). Por ejemplo: "Puedo: 1. Hacer una presentación oral siguiendo un registro (véase Tabla 1). La fiabilidad interna fue $\alpha$ To= .76 y $a$ T1 $=.79$. 
e) Ansiedad ante la defensa oral del TFM. Se utilizaron los mismos 3 ítems que en la escala anterior y con la misma escala de respuesta, pero haciendo referencia al grado de ansiedad que estas situaciones producirían a los alumnos, desde 1 (no me produciría ninguna ansiedad) hasta 5 (me generaría mucha ansiedad). La fiabilidad interna fue $\alpha$ To $=.82$ y a T1= .80 .

f) Rendimiento en exposición oral de TFM. Éste fue evaluado mediante una escala de 10 ítems (ej., adecuada postura corporal) cuyo valor oscila entre o y 1 , y utilizada tanto por el docente para dar retroalimentación (Rendimiento exposición en To y T1) como por los alumnos para realizar la co-evaluación de la presentación de un compañero en To. La calificación final fue la suma de la puntuación obtenida en todos los ítems, sobre una escala de 0 a 5 (Véase Tabla 4).

\section{Procedimiento de recogida y análisis de datos}

Se utilizó un diseño experimental intrasujeto pre-post (To y T1). La variable independiente es la participación en los seminarios, y las variables dependientes son la autoeficacia, nivel de ansiedad y rendimiento en la redacción y defensa oral del TFM. El estudio cuenta con tres fases diferenciadas:

Fase de inicio. Al inicio de curso (condición pre-experimental o To), se realizó una evaluación de la percepción de eficacia y del nivel de ansiedad ante la redacción (Autoeficacia_redacción_To; Ansiedad_redacción_To) y la defensa oral (Autoeficacia_exposición_To; Ansiedad_exposición_To) del TFM del alumnado. A continuación, se indicó a los alumnos que debían realizar un trabajo escrito que les serviría de práctica para su TFM y que, además, sería expuesto públicamente (Simulacro de TFM_To). Para ello, los alumnos recibieron los seminarios 1 y 2 mencionados previamente y, además, se les facilitó la guía del TFM y la rúbrica de evaluación del mismo. En ambos documentos se indican las competencias a adquirir por el alumnado, la forma de evaluación, y en cuánto tiempo debe realizarse el trabajo. Además, se les proporcionó la normativa APA. Este Simulacro de TFM_To debía cumplir con todos los requisitos del TFM que el alumnado debe desarrollar a final de curso, aunque con una extensión significativamente menor (cinco páginas). Además, los participantes debían grabar una exposición oral defendiendo su Simulacro de TFM_To (Exposición_To). Teniendo en cuenta que al alumnado se le pide que su TFM sea defendido sin sobrepasar el tiempo establecido para ello y utilizando soportes visuales, se estableció un tiempo máximo de cinco minutos para dicha grabación, y se pidió a los alumnos se apoyaran en una presentación en powerpoint. Tanto el Simulacro TFM_To como el vídeo de la exposición oral (Exposición_To) debían ser entregados a través de la plataforma Moodle.

Fase de intervención. Una vez finalizada la entrega de estas actividades, los alumnos recibieron formación específica en aspectos relacionados con la redacción de trabajos académicos y con habilidades orales a través de los tres seminarios teórico-prácticos restantes (seminarios 3, 4 y 5). Una vez terminados los seminarios, el alumnado debía:

1) Co-evaluar tanto el Simulacro TFM_To como la Exposición_To de uno de sus compañeros. Las co-evaluaciones se llevaron a cabo a través de Moodle.

2) Recibir retroalimentación del profesor sobre el Simulacro TFM_To (Rendimiento redacción_To) y la Exposición_To (Rendimiento exposición_To). Esta retroalimentación seguía los mismos criterios que las co-evaluaciones.

Fase final. Tras la participación en los seminarios y recibir la retroalimentación de los profesores sobre el Simulacro TFM_To y la Exposición_To, los alumnos rellenaron de nuevo los cuestionarios de nivel de ansiedad y autoeficacia ante la redacción 
(Autoeficacia_redacción_T1; Ansiedad_redacción_T1) y exposición oral (Autoeficacia_exposición_T1; Ansiedad_exposición_T1) del TFM. Además, llevaron a cabo la redacción de un segundo simulacro de TFM (Simulacro TFM_T1) y grabaron una exposición oral del mismo (Exposición_T1) partiendo de la retroalimentación recibida por el profesor y de las habilidades adquiridas en los seminarios. Ambas tareas (Simulacro TFM_T1 y Exposición T1), cuyas pautas para la realización fueron similares a sus homólogos en To (por ej., duración máxima de la exposición de cinco minutos), fueron subidas a la plataforma Moodle.

De los 16 participantes que formaron parte del grupo experimental, uno de ellos no realizó el Simulacro TFM_To por incorporarse tarde al curso académico, mientras que otro no hizo la entrega del Simulacro TFM_T1. Por otro lado, dos participantes no realizaron la Exposición_To ni la Exposición_T1.

Al igual que en la Fase de inicio, los alumnos recibieron retroalimentación del profesor del Simulacro TFM_T1 (Rendimiento redacción_T1) y de la Exposición_T1 (Rendimiento exposición_T1).

Por último, para estudiar si este proyecto de innovación supuso un efecto positivo en la redacción y defensa del TFM, se compararon las calificaciones obtenidas y el número de alumnos presentados en la convocatoria ordinaria del curso 2017/2018 con el curso 2016/2017. Cabe destacar que los tribunales de este Máster actúan cada año con la misma composición de miembros, así como que los directores de TFM no variaron entre ambos cursos.

Todos los análisis se realizaron mediante el programa Prism 5 (GraphPad Software, Inc.). Para estudiar las posibles diferencias estadísticas entre las medias obtenidas en rendimiento, niveles de ansiedad y autoeficacia de los alumnos en To y $\mathrm{T} 1$, tanto en redacción de textos académicos como en la exposición oral, se utilizó una t de student de medidas repetidas. Para analizar las posibles diferencias de medias entre la evaluación realizada por el profesor (retroalimentación en To) y la co-evaluación de los alumnos en To se utilizó una prueba de $t$ de student para muestras independientes. Este mismo análisis se usó para evaluar las posibles diferencias entre las calificaciones de los alumnos en el TFM en el curso 2016/17 (grupo control) y en el 2017/2018 (grupo experimental).

\section{Resultados}

Percepción de autoeficacia y ansiedad ante la redacción y defensa oral del simulacro de TFM: efecto de la participación en el proyecto de innovación docente.

Nuestros resultados muestran la existencia de diferencias estadísticamente significativas entre antes y después de la participación en los seminarios en la autoeficacia de los participantes ante la redacción $\mathrm{t} 15=-2.68, \mathrm{p}<.001$ (Figura $1 \mathrm{~A}$ ) y la exposición oral del Simulacro de TFM t15 = 10.05, $\mathrm{p}<.001$ (Figura 1B). 
A

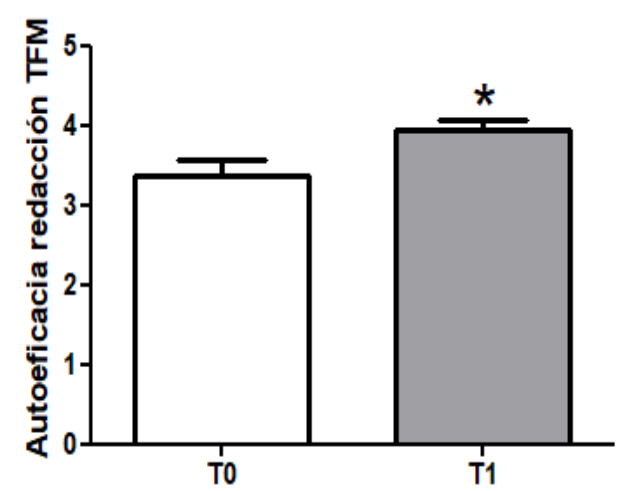

B

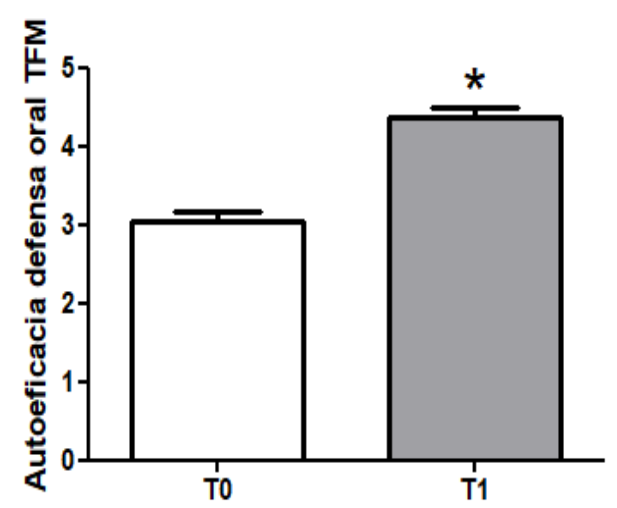

Figura 1. Niveles de autoeficacia ante A. la redacción del TFM y B. la defensa oral del TFM antes y después de la intervención. En la figura se muestran las medias (Me) \pm error estándar de la muestra (SEM) del rendimiento a To y a T1. * $p \leq .05$.

Para entender mejor estas diferencias, se analizó la respuesta de los participantes en cada ítem de las escalas de autoeficacia (Tabla 1).

Tabla 1.

Niveles de autoeficacia de los participantes en cada uno de los ítems antes y después de la intervención. Se muestran Me \pm SEM. * $p \leq .05 y$ **p 0.001.

\begin{tabular}{|c|c|c|c|c|}
\hline & Ítems escala de Autoeficacia & To (Me \pm SEM) & $\begin{array}{l}\text { T1 } \\
(\mathrm{Me} \pm \mathrm{SEM})\end{array}$ & $\begin{array}{l}\text { Estadístico t } \\
\text { student }\end{array}$ \\
\hline \multirow[t]{5}{*}{ Redacción } & " Búsqueda de fuentes bibliográficas & $3.4 \pm 0.18$ & $3.6 \pm 0.20$ & $t_{15}=1.00$ \\
\hline & $\begin{array}{l}\text { Apoyar argumentos en lenguaje } \\
\text { escrito }\end{array}$ & $3.9 \pm 0.17$ & $4.2 \pm 0.16$ & $t_{15}=1.23$ \\
\hline & Manejar la normativa APA & $3.0 \pm 0.36$ & $4.1 \pm 0.19 *$ & $t_{15}=2.57$ \\
\hline & $\begin{array}{l}\text { Escribir y estructurar un texto } \\
\text { científico }\end{array}$ & $3.3 \pm 0.27$ & $3.9 \pm 0.17^{*}$ & $t_{15}=2.18$ \\
\hline & Escribir sin plagio & $3.2 \pm 0.25$ & $3.9 \pm 0.19 *$ & $t_{15}=2.42$ \\
\hline \multirow[t]{3}{*}{$\begin{array}{l}\text { Exposición } \\
\text { oral }\end{array}$} & $\begin{array}{l}\text { Presentación oral siguiendo un } \\
\text { registro científico }\end{array}$ & $3.3 \pm 0.20$ & $4.4 \pm 0.15^{* *}$ & $t_{15}=4.98$ \\
\hline & Ajuste adecuado al tiempo & $3.0 \pm 0.18$ & $4.2 \pm 0.14^{* *}$ & $t_{15}=5.22$ \\
\hline & Contestar adecuadamente al tribunal & $2.8 \pm 0.16$ & $4.6 \pm 0.13^{* *}$ & $t_{15}=10.25$ \\
\hline
\end{tabular}

Por otro lado, la ansiedad de los estudiantes ante la redacción del TFM disminuyó significativamente tras la intervención ( $\mathrm{t} 15=4.18, \mathrm{p}<.001$ ) (Figura $2 \mathrm{~A}$ ), al igual que ocurrió con la ansiedad ante la defensa oral del mismo ( $\mathrm{t} 15=5.97, \mathrm{p}<.001$ ) (Figura $2 \mathrm{~B}$ ). Al analizar cada uno de los ítems de nivel de ansiedad por separado, se observa que se produce una reducción estadísticamente significativa de la ansiedad tras la participación en el programa en todos los aspectos analizados (Tabla 2). 
A

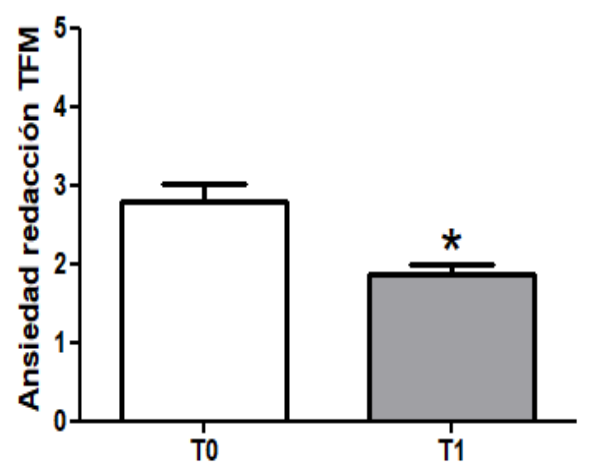

B

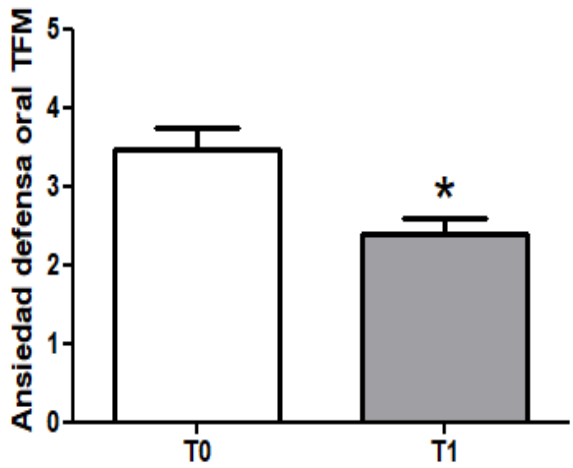

rigura 2. Niveles de ansiedad ante A. Ia readcciurı ueı I rivı y D. Id ueterısd urd ueı

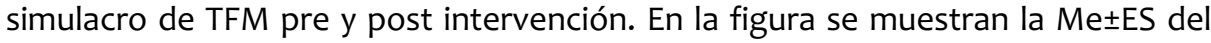
nivel de ansiedad a To y a T1. * $\mathrm{p} \leq .05$.

Tabla 2.

Niveles de ansiedad que produce cada uno de los ítems a los participantes antes y después del proyecto de innovación. Se muestran la Me \pm SEM. * $p \leq .05$ y**p $\leq .001$.

\begin{tabular}{|c|c|c|c|c|}
\hline & Ítems escala de Ansiedad & $\begin{array}{l}\text { To } \\
\text { (Me } \pm \text { SEM) }\end{array}$ & $\begin{array}{l}\text { T1 } \\
(\mathrm{Me} \pm \mathrm{SEM}) \\
\end{array}$ & $\begin{array}{l}\text { Estadístico t } \\
\text { student }\end{array}$ \\
\hline \multirow[t]{5}{*}{ Redacción } & Búsqueda de fuentes & $2.7 \pm 0.27$ & $1.9 \pm 0.14^{*}$ & $t_{15}=2.42$ \\
\hline & $\begin{array}{l}\text { Apoyar argumentos en lenguaje } \\
\text { escrito }\end{array}$ & $2.6 \pm 0.27$ & $1.7 \pm 0.15^{*}$ & $t_{15}=3.22$ \\
\hline & Manejar la normativa APA & $3.1 \pm 0.33$ & $2.0 \pm 0.21 *$ & $t_{15}=3.16$ \\
\hline & $\begin{array}{l}\text { Escribir y estructurar un texto } \\
\text { científico }\end{array}$ & $2.7 \pm 0.28$ & $1.9 \pm 0.19 *$ & $t_{15}=3.50$ \\
\hline & Escribir sin plagio & $2.9 \pm 0.31$ & $1.7 \pm 0.23^{* *}$ & $t_{15}=4.07$ \\
\hline \multirow[t]{3}{*}{$\begin{array}{l}\text { Exposición } \\
\text { oral }\end{array}$} & $\begin{array}{l}\text { Presentación oral siguiendo un } \\
\text { registro científico }\end{array}$ & $3.1 \pm 0.26$ & $2.4 \pm 0.26^{*}$ & $t_{15}=2.66$ \\
\hline & Ajuste adecuado al tiempo & $3.6 \pm 0.27$ & $2.4 \pm 0.27 * *$ & $t_{15}=4.84$ \\
\hline & Contestar adecuadamente al tribunal & $3.7 \pm 0.36$ & $2.4 \pm 0.36 * *$ & $t_{15}=5.84$ \\
\hline
\end{tabular}

Evolución del rendimiento del alumnado en la redacción del simulacro de TFM y la defensa oral.

Los resultados muestran que el rendimiento en la redacción del Simulacro de TFM mejora significativamente tras la participación en el proyecto ( $\mathrm{t} 12=5.04, \mathrm{p}<.01$ ) (Figura 3A). Además, los resultados indican que las diferencias entre las medias otorgadas por el profesor (Rendimiento redacción_To) y las otorgadas por los alumnos (co-evaluación) no son estadísticamente significativas, siendo t24=1.25, $\mathrm{p}=.22$ (Figura 3B).

Para profundizar en los resultados anteriores, se analizaron los resultados obtenidos en cada uno de los ítems relacionados con el rendimiento en la redacción del Simulacro de TFM en To y T1 (Ver Tabla 3). Se observaron mejoras significativas en el uso adecuado de la normativa APA y en la redacción del trabajo científico. 
A

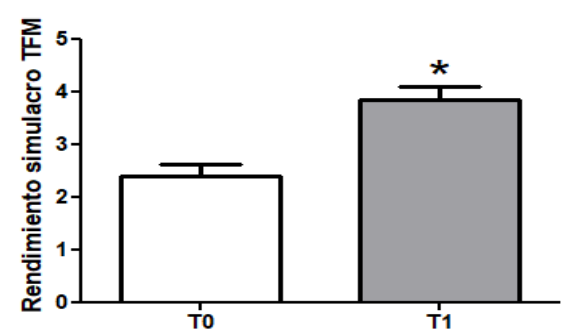

B

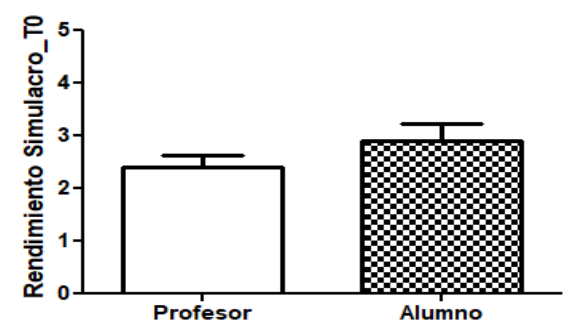

Figura 3. Me \pm ES del Rendimiento_redacción A. en el TFM_To y en T1 y B. del rendimiento_redacción_TFM_To cuando este fue evaluado por el profesor o por los propios alumnos (coevaluación). * $\mathrm{p} \leq .05$.

Tabla 3.

Rendimiento en el Simulacro TFM en cada uno de los ítems antes y después del proyecto de innovación. ${ }^{*} p \leq .05 y^{* *} p \leq .001$.

\begin{tabular}{llll}
\hline \hline Ítems escala de Rendimiento redacción & To (Me \pm SEM) & T1 (Me \pm SEM) & $\begin{array}{l}\text { Estadístico } t \\
\text { student }\end{array}$ \\
\hline \hline Citas que cumplen APA & $0.28 \pm 0.11$ & $0.66 \pm 0.12^{*}$ & $\mathrm{t}_{12}=2.17$ \\
Referencias que cumplen APA & $0.11 \pm 0.18$ & $0.48 \pm 0.13^{*}$ & $\mathrm{t}_{12}=2.62$ \\
Todas las citas aparecen en referencias & $0.43 \pm 0.14$ & $0.93 \pm 0.07^{*}$ & $\mathrm{t}_{12}=3.20$ \\
Todas las referencias se citan en el texto & $0.29 \pm 0.12$ & $0.86 \pm 0.10^{*}$ & $\mathrm{t}_{12}=3.74$ \\
Escribir sin plagio & $0.78 \pm 0.8$ & $0.93 \pm 0.07$ & $\mathrm{t}_{12}=1.48$ \\
No usar exceso de citas textuales & $0.71 \pm 0.12$ & $0.93 \pm 0.07$ & $\mathrm{t}_{12}=1.48$ \\
Redacción en lenguaje científico & $0.54 \pm 0.09$ & $0.75 \pm 0.08^{*}$ & $\mathrm{t}_{12}=2.50$ \\
Bibliografía suficiente & $0.57 \pm 0.14$ & $0.64 \pm 0.13$ & $\mathrm{t}_{12}=1.00$ \\
Bibliografía de calidad & $0.57 \pm 0.14$ & $0.71 \pm 0.12$ & $\mathrm{t}_{12}=1.48$ \\
\hline \hline
\end{tabular}

Nota: Las puntuaciones de cada uno de los ítems oscilan entre o y 1. Los ítems 1 y 2 puntúan o si hay más de dos errores, 0.5 si hay un error y 1 si no hay errores. Los demás ítems puntúan o si la respuesta es negativa y 1 si la respuesta es afirmativa.

Por otro lado, el análisis de los vídeos de las exposiciones orales por parte del docente muestra que la calidad de la Exposición oral de TFM mejora significativamente tras la participación en el programa ( $\mathrm{t} 12=4.53, \mathrm{p}<.01)$ (Figura $4 \mathrm{~A}$ ). Además, no existen diferencias estadísticamente significativas entre la evaluación realizada por el profesor (Rendimiento exposición_To) y por el alumnado (Co-evaluación exposición_To), siendo t24= 0.16, p = .86 (Figura 4B). En la Tabla 4 se muestran los resultados tras analizar cada uno de los ítems relativos al rendimiento en la exposición oral. Se observaron diferencias significativas antes y después de la participación en el proyecto en el uso adecuado de la presentación, en el tono de voz, la expresión facial y el uso de marcadores no verbales. 
A

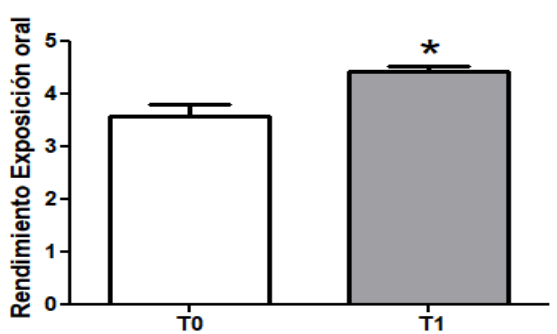

B

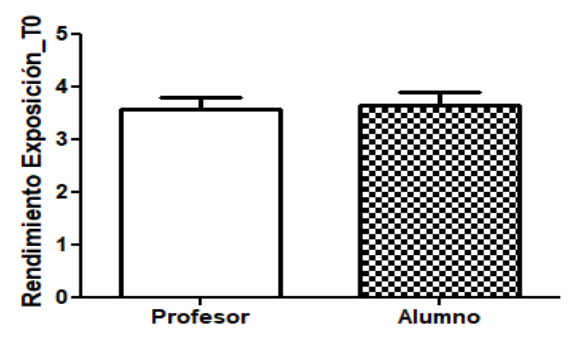

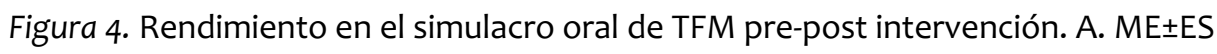
del rendimiento To y a T1. B. Me \pm ES del rendimiento del simulacro del TFM cuando este fue evaluado por el profesor o por los propios alumnos (coevaluación). ${ }^{*} \mathrm{p} \leq .05$.

Tabla 4

Rendimiento en el Simulacro de exposición oral del TFM en cada uno de los ítems antes y después del proyecto de innovación. * $p \leq .05 y * * p \leq .001$.

\begin{tabular}{llll}
\hline \hline Ítems escala de Rendimiento & To (Me \pm SEM) & T1 (Me \pm SEM) & Estadístico t student \\
\hline \hline Postura adecuada & $0.81 \pm 0.09$ & $0.96 \pm 0.04$ & $t_{12}=1.48$ \\
Uso de marcadores no verbales & $0.62 \pm 0.08$ & $0.85 \pm 0.07^{*}$ & $t_{12}=3.21$ \\
Expresión facial agradable dirigida al interlocutor & $0.77 \pm 0.07$ & $1.00 \pm 0.00^{*}$ & $t_{12}=3.21$ \\
Tono de voz y ritmo de habla adecuado & $0.77 \pm 0.07$ & $1.00 \pm 0.00^{*}$ & $t_{12}=3.21$ \\
Respeto del tiempo establecido para la exposición & $0.77 \pm 0.12$ & $1.00 \pm 0.00$ & $t_{12}=1.90$ \\
Uso de un léxico adecuado a la situación comunicativaa & $0.81 \pm 0.07$ & $0.92 \pm 0.05$ & $t_{12}=1.39$ \\
Uso de un registro académico y formal & $0.62 \pm 0.08$ & $0.73 \pm 0.11$ & $t_{12}=1.00$ \\
Discurso ordenado & $0.73 \pm 0.07$ & $0.77 \pm 0.07$ & $t_{12}=0.43$ \\
Uso adecuado y pertinente de los conectores & $0.58 \pm 0.11$ & $0.62 \pm 0.08$ & $t_{12}=0.32$ \\
Uso pertinente de la presentación & $0.69 \pm 0.09$ & $1.00 \pm 0.00^{*}$ & $t_{12}=3.41$ \\
\hline \hline
\end{tabular}

Nota: La puntuación que obtuvo cada estudiante en cada uno de los ítems fue entre o y 1 siendo 1 (óptimo), 0.5 (adecuado) y o (inadecuado).

\section{Efectos del proyecto de innovación docente en la calidad del TFM y su defensa oral ante el tribunal.}

Nuestros datos muestran que son más los alumnos que defendieron su TFM en la primera convocatoria en el curso en el que se realizó el presente proyecto (el 62,5\%) (2017/2018) en comparación con el grupo control (46,1\%). Por otro lado, al comparar las calificaciones obtenidas en la asignatura TFM en estos dos cursos, éstas fueron significativamente mejores en los alumnos que participaron en el proyecto de innovación docente $(M=8.1$, ES $=0.36)$ respecto a los que no $(M=7.1, E S=0.31)$, siendo $t 24=2.24 p \leq .05$.

\section{Discusión y conclusiones}

Diversos trabajos hacen referencia a la escasa preparación de los alumnos universitarios y postgraduados para redactar trabajos académicos (Bhargava, 2015; Granello, 2001; Lloyd, 2007) y enfrentarse a defensas orales que serán evaluadas por un tribunal (Onieva-López, 
2016). Este problema está también presente en los alumnos de Máster de profesorado de nuestra Universidad. Para abordarlo, se presenta un trabajo que evalúa la eficacia de un proyecto de innovación docente cuyo objetivo principal es el de aumentar el rendimiento de los alumnos en el TFM, mejorar su autoeficacia y disminuir su ansiedad. Para ello se impartieron 5 seminarios teórico-prácticos acompañados de un proceso de entrenamiento en el que los alumnos debían redactar y corregir posteriormente un "Simulacro de TFM" y un vídeo grabado de la "Exposición oral” del mismo.

Los datos obtenidos muestran que los participantes presentan un bajo nivel de autoeficacia y un alto nivel de ansiedad al inicio del curso académico cuando piensan en enfrentarse a la redacción y defensa del TFM. Sin embargo, la participación en el proyecto de innovación docente logra que estos niveles de ansiedad disminuyan y la autoeficacia aumente de forma significativa. Es más, el rendimiento de los alumnos tanto en la redacción de trabajos académicos como en habilidades comunicativas también mejora significativamente $y$, además, los hace capaces de evaluar con éxito a sus compañeros en estas actividades.

De forma específica, cuando analizamos la variable ansiedad, esta disminuye significativamente en todos los aspectos estudiados tanto en la redacción del trabajo (plagio, redacción científica, uso de normativa APA, búsqueda de bibliografía, etc.) como en las competencias orales (uso de un registro adecuado, ajuste del tiempo y respuesta al tribunal), tras recibir los seminarios y realizar y corregir el "Simulacro TFM" y la "Exposición oral". Estos resultados se encuentran apoyados por el estudio de Culler y Holaham (1980) en el que los autores consideran que el entrenamiento es fundamental para disminuir la ansiedad en los estudiantes ante una determinada tarea. En cuanto a la autoeficacia, a nivel escrito se incrementa significativamente en los ítems que hacen referencia al uso de la normativa APA, escribir y estructurar un texto científico y no cometer plagio. Sin embargo, no se observaron diferencias significativas en la búsqueda fuentes bibliográficas con rigor científico ni en la síntesis e integración de ideas obtenidas de distintasfuentes bibliográficas para apoyar argumentos.

En relación al rendimiento en la redacción del Simulacro de TFM, se observa una mejora significativa tanto en el manejo de la normativa APA como en la redacción en un lenguaje científico. Sin embargo, no se producen mejoras en disminuir el uso de citas textuales, en evitar cometer plagio, ni en el uso de una bibliografía adecuada.

Por un lado, observamos que rendimiento y autoeficacia siguen un patrón similar. En ambos se producen aumentos significativos en el uso adecuado de la normativa APA y en la redacción del trabajo científico. Estos resultados muestran que la autoeficacia es selectiva de la tarea (Bandura, 1986), ya que los estudiantes se sienten autoeficaces para usar la normativa APA pero no para buscar bibliografía relevante. Además, autoeficacia y rendimiento parecen estar íntimamente relacionados, tal y como se describe en estudios previos de relevancia (Doménech-Betoretet al. 2017; Galleguillos, 2017; Lane y Lane, 2001; Pajares, 2001). De esta manera, los participantes se sienten autoeficaces para usar la normativa APA y además aumentan su rendimiento en esta tarea. Sin embargo, nuestros resultados muestran que no se produce una mejora significativa en la búsqueda de fuentes bibliográficas. Esto puede ser debido a que los seminarios se centraron más en distinguir qué es bibliografía de calidad y los índices de calidad existentes que en la búsqueda propiamente dicha. Se tomó esta decisión porque muchos participantes usaron para la redacción de su Simulacro TFM_To referencias de dudoso rigor científico, tales como blogs, vídeos de youtube, TFGs y TFMs previos como única bibliografía o como bibliografía mayoritaria. 
A pesar de que se sienten significativamente más eficaces, los estudiantes de Máster no mejoraron significativamente en evitar el plagio y el uso de citas textuales. Esto puede deberse a que los alumnos prácticamente no cometieron plagio en el Simulacro de TFM_To, ni tampoco usaron numerosas citas textuales.

En cuanto a las competencias comunicativas, la participación en el proyecto de innovación aumentó significativamente la autoeficacia de los alumnos en la realización de una presentación oral siguiendo un registro científico, en el ajuste al tiempo establecido y en contestar adecuadamente las preguntas de los miembros del tribunal. Al igual que sucede con competencias escritas, se observan ciertos paralelismos entre autoeficacia y rendimiento en competencias orales (Hirsch et al. 2006). De este modo, los alumnos mejoraron significativamente su rendimiento en el uso pertinente de la presentación powerpoint, en el uso de marcadores no verbales, en el tono y ritmo del habla y en la expresión facial adecuada. En general, los estudiantes manejaron correctamente el tiempo otorgado para la exposición en el To, lo cual podría explicar por qué no se obtuvieron diferencias significativas en este ítem entre To y T1. A esto hay que añadir que, al carecer de tribunal, no se pudo evaluar cómo se desenvuelven al responder al mismo y si se producían mejoras significativas entre To y T1.

Por último, hemos observado que los alumnos del grupo experimental obtuvieron una nota significativamente superior en la asignatura TFM respecto a los alumnos del curso anterior (grupo control). A pesar de las diferencias individuales que puedan existir entre los alumnos de ambos grupos, los tutores de TFM fueron los mismos en ambos cursos y los tribunales estuvieron compuestos por los mismos profesores. Además, no existen diferencias significativas en las calificaciones obtenidas en la asignatura que imparten los autores de este trabajo entre los estudiantes de cada uno de los grupos. A esto hay que añadir que el número de alumnos que presentaron su TFM en la convocatoria de junio fue mayor en el grupo experimental que en el control. Estos resultados sugieren que, a pesar de la dificultad que la redacción y defensa del TFM puede tener para los alumnos, un aumento de su percepción de eficacia en estas tareas les ayuda a no evitar situaciones difíciles (Roses y Humanes, 2014) y, por tanto, a presentar su TFM en la primera convocatoria.

Los resultados son positivos y alentadores. Sin embargo, a la hora de interpretarlos es preciso tener en consideración algunas limitaciones del estudio. En primer lugar, el reducido número de participantes no permite la generalización de los resultados. Futuros trabajos con una muestra mayor y más diversa ayudarán a establecer la eficacia del proyecto de innovación docente. En segundo lugar, no existen escalas para registrar los niveles de autoeficacia y ansiedad ante tareas específicas y necesarias para la superación del TFM. Los instrumentos aquí utilizados se basan en escalas precedentes y han resultado tener buena consistencia interna. No obstante, convendría validar dichos instrumentos de cara a ser utilizados en próximos trabajos. En tercer lugar, se ha registrado la autoeficacia y nivel de ansiedad del alumnado ante la situación de responder a preguntas del tribunal, pero no se ha podido medir si su competencia en esta tarea ha mejorado realmente. Futuros trabajos podrían incluir un tribunal ante el que presentar la exposición oral del simulacro de TFM, de manera que pueda evaluarse la destreza de los estudiantes ante esta tarea de manera más objetiva.

En conclusión, nuestros resultados muestran que recibir formación específica a través de seminarios teórico-prácticos aumenta los niveles de autoeficacia y el rendimiento del alumnado tanto en la exposición oral como en la redacción de trabajos académicos. En concordancia con estudios previos, la autoeficacia parece influir tanto en las competencias escritas (Callinan et al. 2018; Pajares y Johnson 1996) como orales (Hirsch et al. 2006). Además, en línea con García-López, Díez-Bedmar y Almansa-Moreno (2013), la formación 
obtenida por los alumnos en los seminarios dio lugar a una disminución de sus niveles de ansiedad al enfrentarse a la redacción y defensa del TFM. Parece necesario trabajar en estudios futuros aquellos aspectos en los que no se han observado cambios significativos, como por ejemplo en la búsqueda de bibliografía científica relevante. La necesidad de redacción de textos científicos, así como de poseer buenas habilidades de comunicación es relevante en la mayoría de grados y postgrados de la universidad. Por ello, consideramos que proyectos de innovación docente como el aquí presentado son relevantes para el proceso de enseñanza-aprendizaje de los estudiantes. Además, en el caso del Máster de Profesorado, las habilidades de redacción y, sobre todo, las orales adquiridas durante el proyecto los prepara para su futura labor como docentes.

\section{Referencias}

Bandura, A. (1986). Social Foundations of Thoughts and Action. A social cognitive theory. Englewood Cliffs, NJ: Prentice Hall.

Bandura, A. (2006). Guide for constructing self-efficacy scales. En F. Pajares y T. Urdan (Ed.), Self-efficacy beliefs of adolescents (Vol. 5, pp. 307-337). Greenwich, CT: Information Age Publishing.

Bartlett, A. y Mercer, G. (2000). Re-conceptualising discourses of power in postgraduate pedagogies. Teaching in Higher Education, 5 (2), 195-204. DOI: https://doi.org/10.1080/135625100114849.

Bazrafkan, L., Shokrpour, N., Yousefi, A. y Yamani, N. (2016). Management of Stress and Anxiety AmongPh.D Students During Thesis Writing: A Qualitative Study. HealthCare Manager, 35, 231-40. DOI: 10.1097/HCM.0000000000000120.

Becerra Traver, M.T. (2017). La habilidad de hablar en público. Una experiencia formativa con estudiantes universitarios. Revista Electrónica Interuniversitaria de Formación del Profesorado, 20 (3), 117-129. DOI:

http://dx.doi.org/10.6018/reifop.20.3.270631

Bhargava, P. (2015). The KISS Principle in Academic Writing. CurrProblDiagnRadiol, 44 (6): 473. DOI: 10.1067/j.cpradiol.2015.08.005.

Blanco, A. (Coord.) (2009). Desarrollo y evaluación de competencias en educación superior. Madrid: Narcea.

Callinan, C.J., van der Zee, E. y Wilson, G. (2018). Developing essay writing skills: an evaluation of the modeling behaviour method and the influence of student self-efficacy. Journal of further and higher education, 42 (5), 608-622. DOI: https://doi.org/10.1080/0309877X.2017.1302564.

Castelló, M. e Iñesta, A. (2012). Texts as artifacts-in-activity: Developing authorial identity and academic voice in writing academic research papers. En M. Castelló y C. Donahue (Ed.), University writing: Selves and texts in academic societies (pp. 177-200). Londres: Emerald Group.

Chan, V. (2011). Teaching oral communication in undergraduate science: Are we doing enough and doing it right? Journal of Learning Design, 4, 71-79. DOI: http://dx.doi.org/10.5204/jld.v4i3.82.

Contreras, F., Espinosa, J. C., Esguerra, G., Haikal, A., Polanía, A. y Rodríguez, A. (2005). Autoeficacia, ansiedad y rendimiento académico en adolescentes. Perspectivas en Psicología, 2, 184-194. 
Culler, R. E. y Holahan, C. J. (1980). Test anxiety and academic performance: the effects of study-related behaviors. Journal of Educational Psychology, 72, 16-20. DOI: 10.1037/00220663.72.1.16.

Doménech-Betoret, F., Abellán-Roselló, L. y Gómez-Artiga, A. (2017). Self-Efficacy, Satisfaction, and Academic Achievement: The Mediator Role of Students' ExpectancyValue Beliefs, Frontiers in Psychology, 8, 1193. DOI: 10.3389/fpsyg.2017.01193.

Galleguillos, P. (2017). Autoeficacia académica y rendimiento escolar: un estudio metodológico y correlacional en escolares. Reidocrea, 6, 156-169.

García-López, L. J., Díez-Bedmar, B. M. y Almansa-Moreno, J. M. (2013). From Being a Trainee to Being a Trainer: Helping Peers Improve their Public Speaking Skills Revista de Psicodidáctica, 18 (2), 331-342. DOI: 10.1387/RevPsicodidact.6419.

Granello, D. H. (2001). Promoting cognitive complexity in graduate written work; Using Bloom's taxonomy as a pedagogical tool to improve literature reviews. Counselor Education and Supervision, 40, 292-307. DOI: https://doi.org/10.1002/j.15566978.2001.tb01261.x

Hirsch, C. R., Mathews, A., Clark, D. M., Williams, R. y Morrison, J. A. (2006). The causal role of negative imagery in social anxiety: a test in confident public speakers. Journal of BehaviorTherapy and ExperimentalPsychiatry, 37, 159-70. DOI: 10.1016/j.jbtep.2005.03.003.

Jato Seijas, E., Cajide Val, J., García Antelo, B. y Zamora Rodríguez, E.T. (2018).Percepciones del profesorado universitario sobre los procesos de organización y tutorización de los Trabajos Fin de Grado. Revista Electrónica Interuniversitaria de Formación del Profesorado, 21(3), 75-91. DOI: http://dx.doi.org/10.6018/reifop.21.3.332051

Lane, J., y Lane, A. M. (2001). Self-efficacy and academic performance. Social Behavior and Personality, 29, 687-694. DOI: 10.2224/sbp.2001.29.7.687.

Lloyd, M (2007). Developing academic writing skills: the PROCESS framework. Nurs Stand, 21, 50-6. DOI: 10.7748/ns2007.06.21.40.50.c4572.

Onieva-López, J.L. (2016). Estrategias didácticas y recomendaciones para la defensa oral de los Trabajos Fin de Grado y Fin de Máster. Revista Electrónica Interuniversitaria de Formación del Profesorado, 19, 185-198. DOI: https://doi.org/10.6018/reifop.19.3.206331.

Orejudo, S., Sánchez, M. T. y Briz, E. (2012). Resultados de un programa para reducir el miedo y aumentar la autoeficacia para hablar en público en estudiantes universitarios de primer año. Estudiossobreeducación 22, 199-217.

Pajares, F. (2001).Self-efficacy beliefs in academic settings. Review of Educational Research, 66(4), 543-578. DOI: https://doi.org/10.3102/00346543066004543

Pajares, F. y Johnson, M. J. (1996). Self-Efficacy Beliefs in the Writing of High-School Students: A Path Analysis. Psychology in the Schools, 33, 163-175.

Palatini, P., Bratti, P., Palomba, D., Bonso, E., Saladini, F., Benetti, E. y Casiglia E. (2011). BP reactivity to public speaking in stage 1 hypertension: influence of different task scenarios. Blood Press, 20, 290-295. DOI: 10.3109/08037051.2011.566011.

Pelias, R. J. (2003). The academic tourist: an autoethnography. Qualitative Inquiry, 9 (3), 369-373. DOI: https://doi.org/10.1177/1077800403009003003

Pervin, L. (1997). La ciencia de la personalidad. Madrid: McGraw-Hill.

Roses, S. y Humanes, M. L. (2014). La innovación educativa, subestimada: Análisis de la importancia que le otorgan los estudiantes de Periodismo. Historia y Comunicación Social, 19, 479-490. DOI: http://dx.doi.org/10.5209/rev_HICS.2014.v19.45148 
Sánchez, M. E., Rosales, E. y Cañedo, I. (1994). El discurso expositivo: una comparación entre profesores principiantes y novatos. Infancia y Aprendizaje, 67, 51-74. DOI: https://doi.org/10.1174/021037094321268868.

Sanjuán, M., Senís, J. y Del Moral, C. (2017). Diseño y evaluación de un proceso de formación en habilidades comunicativas para la docencia. Porta Linguarum, monográfico, 2, 57-71.

Tejedor Tejedor, F.J. (2018). Investigación educativa: la utilidad como criterio social de calidad. Revista de Investigación Educativa, 36 (2), 315-330. DOI: http://dx.doi.org/10.6018/rie.36.2.326311. 